\title{
Phagocytic receptors activate and immune inhibitory receptor SIRP $\alpha$ inhibits phagocytosis through paxillin and cofilin
}

\section{Miri Gitik $^{1+}{ }^{1}$, Rachel Kleinhaus ${ }^{1}$, Smadar Hadas ${ }^{1}$, Fanny Reichert ${ }^{1}$ and Shlomo Rotshenker ${ }^{1,2}$ *}

${ }^{1}$ Department of Medical Neurobiology, Institute for Medical Research Israel-Canada, Faculty of Medicine, Hebrew University, Jerusalem, Israel

${ }^{2}$ Brain Disease Research Center, Institute for Medical Research Israel-Canada, Faculty of Medicine, Hebrew University, Jerusalem, Israel

\section{Edited by:}

Raquel Ferreira, University of

Southern California, USA

\section{Reviewed by:}

Uwe-Karsten Hanisch, University of Göttingen, Germany

Raquel Ferreira, University of

Southern California, USA

${ }^{*}$ Correspondence:

Shlomo Rotshenker, Department of Medical Neurobiology, Institute for Medical Research Israel-Canada, Faculty of Medicine, Hebrew University, Ein-Kerem, P.O.Box 12272, Jerusalem 91120, Israel e-mail: shlomor@ekmd.huji.ac.il

${ }^{\dagger}$ Present address:

Miri Gitik, Laboratory of Neurogenetics, NIH/NIAAA, 5625

Fishers Lane, Rockville, MD 20892-9412, USA
The innate immune function of phagocytosis of apoptotic cells, tissue debris, pathogens, and cancer cells is essential for homeostasis, tissue repair, fighting infection, and combating malignancy. Phagocytosis is carried out in the central nervous system (CNS) by resident microglia and in both CNS and peripheral nervous system by recruited macrophages. While phagocytosis proceeds, bystander healthy cells protect themselves by sending a "do not eat me" message to phagocytes as CD47 on their surface ligates immune inhibitory receptor SIRP $\alpha$ on the surface of phagocytes and SIRP $\alpha$ then produces the signaling which inhibits phagocytosis. This helpful mechanism becomes harmful when tissue debris and unhealthy cells inhibit their own phagocytosis by employing the same mechanism. However, the inhibitory signaling that SIRP $\alpha$ produces has not been fully revealed. We focus here on how SIRPa inhibits the phagocytosis of the tissue debris "degenerated myelin" which hinders repair in axonal injury and neurodegenerative diseases. We tested whether SIRP $\alpha$ inhibits phagocytosis by regulating cytoskeleton function through paxillin and cofilin since (a) the cytoskeleton generates the mechanical forces that drive phagocytosis and (b) both paxillin and cofilin control cytoskeleton function. Paxillin and cofilin were transiently activated in microglia as phagocytosis was activated. In contrast, paxillin and cofilin were continuously activated and phagocytosis augmented in microglia in which SIRP $\alpha$ expression was knocked-down by SIRP $\alpha$-shRNA. Further, levels of phagocytosis, paxillin activation, and cofilin activation positively correlated with one another. Taken together, these observations suggest a novel mechanism whereby paxillin and cofilin are targeted to control phagocytosis by both the activating signaling that phagocytic receptors produce by promoting the activation of paxillin and cofilin and the inhibiting signaling that immune inhibitory SIRP $\alpha$ produces by promoting the inactivation of paxillin and cofilin.

Keywords: microglia, macrophage, phagocytosis, CD47, SIRP $\alpha$, paxillin, cofilin, cytoskeleton

\section{INTRODUCTION}

Phagocytosis, the engulfment and internalization of large particles by phagocytes, is an innate immune function which is carried out in the central nervous system (CNS) parenchyma by resident microglia and in both CNS parenchyma and peripheral nervous system (PNS) nerves by recruited bone marrow-derived macrophages. Phagocytosis is essential for homeostasis as it clears apoptotic and aging cells, tissue repair when it removes tissue debris, fighting infection since it scavenges pathogens and combating malignancy while it eliminates cancer cells. During such phagocytosis, bystander healthy cells are protected from being phagocytosed. CD47-SIRP $\alpha$ interactions provide this protection when CD47 on the surface of healthy cells binds and activates the immune inhibitory receptor signal regulatory protein- $\alpha$ (SIRP $\alpha$; also known as CD172 $\alpha$ and SHPS-1) on the surface of phagocytes and SIRP $\alpha$ then produces the signaling that inhibits phagocytosis (Barclay and Brown, 2006; Matozaki et al., 2009; Oldenborg, 2013). Such observations led to define CD47 as a marker of "self" which sends a "do not eat me" message to phagocytes (Oldenborg et al., 2000).

This protective mechanism becomes detrimental under pathological conditions when tissue debris (Gitik et al., 2011) and malignant cells (Chao et al., 2012; Kim et al., 2012) employ CD47SIRP $\alpha$ interactions to inhibit their own clearance. We (Gitik et al., 2011) and others thereafter (Han et al., 2012) documented in this regard that the tissue debris "degenerated myelin" inhibits its own phagocytosis as CD47 on degenerated myelin ligates $\operatorname{SIRP} \alpha$ on macrophages and microglia. Intact myelin is a specialized extension of Schwann cells in the PNS and oligodendrocytes in the CNS, and further, it surrounds the larger diameter of PNS and CNS axons, enabling them fast conduction of electrical activity. Myelin breaks down in Wallerian degeneration following traumatic axonal injury (Waller, 1850; Vargas and Barres, 2007; Rotshenker, 2011) and in neurodegenerative diseases such as multiple sclerosis (Stadelmann and Bruck, 2008). The rapid phagocytosis of the tissue debris degenerated myelin so 
produced is essential since it impedes repair and exacerbates disease by arresting the regeneration of severed adult axons (David and Aguayo, 1981), preventing remyelination (Kotter et al., 2006) and advancing the production of membrane attack complexes that damage nearby intact tissue (Mead et al., 2002). Thus, understanding how SIRP $\alpha$ inhibits the phagocytosis of degenerated myelin is of utmost importance.

Previous studies documented that $\operatorname{SIRP} \alpha$ signaling involves the recruitment of tyrosine phosphatases SHP-1 and SHP-2 to the cytoplasmic domain of SIRP $\alpha$, and subsequently, SHP- 1 and SHP-2 may dephosphorylate phosphotyrosine sites in their immediate downstream target molecules (Barclay and Brown, 2006; Matozaki et al., 2009; Oldenborg, 2013). However, the molecular events further downstream to the SIRP $\alpha /$ SHP-1/2 complex have not been fully elucidated.

The phagocytosis of degenerated myelin is mediated by phagocytic receptors complement receptor-3 (CR3), scavenger receptor SRA-I/II (SRA) and Fc $\gamma$ receptor $(\mathrm{Fc} \gamma \mathrm{R})$ (Reichert et al., 2001; Reichert and Rotshenker, 2003; Rotshenker, 2003). CR3 ligates both unopsonized and C3bi-opsonized degenerated myelin (i.e., opsonized by complement protein C3bi), SRA ligates unopsonized degenerated myelin, and $\mathrm{F} \gamma \mathrm{R}$ ligates IgG-opsonized degenerated myelin (i.e., opsonized by anti-myelin antibodies). Further, degenerated myelin can simultaneously ligate several receptors. We focus in this study on the cytoskeleton as a potential target through which SIRP $\alpha$ may inhibit the phagocytosis of degenerated myelin which is mediated mostly by CR3, to a lesser level by SRA, and not at all by Fc $\gamma \mathrm{R}$ since phagocytosis was assayed in the absence of anti-myelin antibodies.

We previously documented that CR3 and SRA mediated phagocytosis of degenerated myelin involves structural changes in phagocytes. First, filopodia and lamellipodia extend and engulf the myelin-debris, and then, filopodia/lamellipodia retract, and pull-in the myelin-debris into the phagocyte where it is degraded (Hadas et al., 2012). Extension and retraction of membrane protrusions are driven by mechanical forces that are generated by the cytoskeleton. The production of membrane protrusions depends on the remodeling of filamentous actin (F-actin); i.e., the breakdown of old filaments and the production of new ones (Wang etal., 2007; Bernstein and Bamburg, 2010). Cofilin/ADF (actin depolymerizing factor) is a family of proteins that controls F-actin remodeling. Active unphosphorylated cofilin advances the remodeling F-actin and thereby the production of filopodia/lamellipodia whereas inactive phosphorylated cofilin (p-cofilin) stabilizes F-actin and thereby reduces the production of filopodia/lamellipodia. We previously documented in this regard that (a) filopodia/lamellipodia are involved in the phagocytosis of degenerated myelin (Hadas et al., 2012); (b) active unphosphorylated cofilin advances whereas inactive p-cofilin reduces phagocytosis (Hadas et al., 2012); (c) the small GTPase RhoA, which signals through ROCK, stabilizes F-actin, and further down-regulates phagocytosis (Gitik etal., 2010). The retraction of filopodia/lamellipodia can be driven by mechanical forces that are generated by contraction which is based on the interaction between F-actin and nonmuscle-myosin in which motor activity was triggered (Clark etal., 2007; Vicente-Manzanares etal., 2009). We previously documented in this regard that the phagocytosis of degenerated myelin is advanced by myosin light chain kinase (MLCK) after it triggers motor activity in non-muscle-myosin which then leads to F-actin/non-muscle-myosin-based contraction (Gitik et al., 2010).

Taken that (a) active cofilin advances the phagocytosis of degenerated myelin by CR3 (an $\alpha \mathrm{M} / \beta 2$ integrin; Hadas et al., 2012), (b) integrins promote the activation the scaffold/adaptor protein paxillin through focal-adhesion-kinase (FAK) and Src and, (c) paxillin in its active phosphorylated state (p-paxillin) can indirectly activate cofilin (Deakin and Turner, 2008), we hypothesize that SIRP $\alpha$ could inhibit phagocytosis by promoting the inactivation of both paxillin and cofilin (Figure 1). Observations made in this study support this working hypothesis, further suggesting that phagocytosis is determined by the balance between CR3 and SRA advancing phagocytosis by promoting paxillin and cofilin activation and SIRP $\alpha$ inhibiting phagocytosis by promoting paxillin and cofilin inactivation.

\section{MATERIALS AND METHODS ANIMALS}

Animals Balb/C mice that were obtained from Harlan (Israel) were handled in accordance with the national research council guide for the care and use of laboratory animals and the approval of the institutional committee.

\section{PRIMARY MICROGLIA}

Primary microglia were isolated from brains of neonate mice as previously described (Reichert and Rotshenker, 2003). In brief, brains were stripped of their meninges, enzymatically dissociated, cells plated on poly-L-lysine coated flasks for 1 week, replated for 1-2 h on bacteriological plates and non-adherent cells washed away. The vast majority (over 95\%) of adherent cells are microglia judged by morphology and positive immunoreactivity to Galectin3/MAC-2, CR3, and F4/80 (Reichert and Rotshenker, 1996, 1999)

\section{GENERATION OF MICROGLIA WITH STABLE REDUCED SIRP $\alpha$ EXPRESSION}

Generation of microglia with stable reduced SIRP $\alpha$ expression was previously described and documented by us (Gitik et al., 2011). Reduction of SIRP $\alpha$ expression was achieved through lentiviral infection of wild-type Balb/C microglia with short hairpin RNAs directed against mouse SIRP $\alpha$ mRNA (SIRP $\alpha$-shRNA) using pLKO.1 puro plasmids (Sigma, Israel). Three different shRNA sequences were used. All were effective in reducing $\operatorname{SIRP} \alpha$ expression $(60,70$, and $90 \%$ of levels in wild-type microglia). The one used in this study, as in our previous study (Gitik et al., 2011), was the SIRP $\alpha$ cDNA coding sequence 5'CCGGTGGTTCAAAGAACTCGAGTTCTTGCCCATCTTTGAACCATTTTTG-3'. The plasmid was transfected into a 293T-based packaging cell line, and the resulting culture supernatant was used for lentiviral infection. Infected microglia were selected on the basis of their resistance to puromycin brought by the pLKO.1 plasmid. Levels of $\operatorname{SIRP} \alpha$ protein expression were monitored by immuno blot analysis. As a control, microglia were infected in a similar way with the shRNA sequence 5'CTTACGCTGAGTACTTCGA-3' against the non-target firefly Luciferase gene. We refer to these microglia 


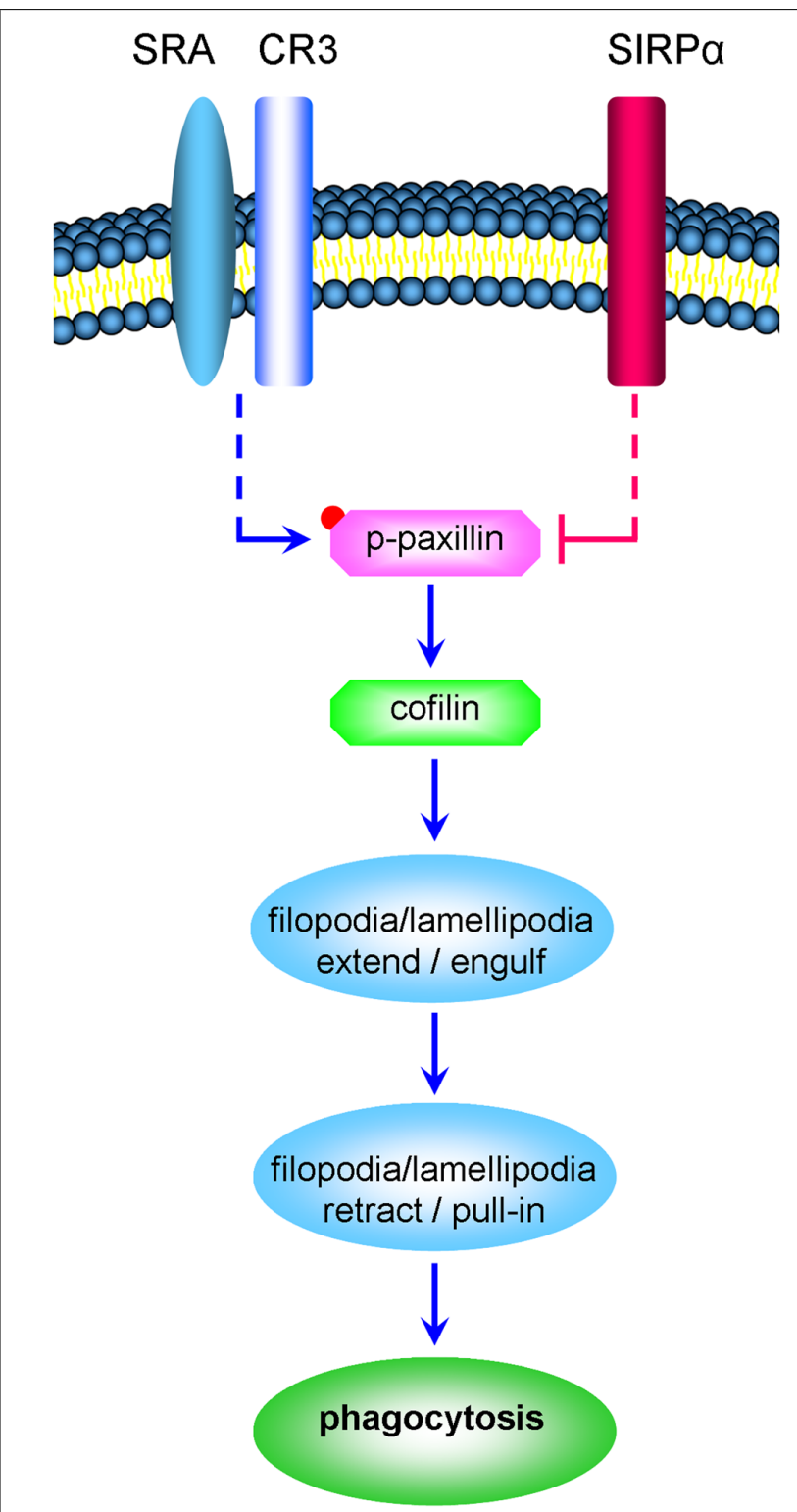

FIGURE 1 | CR3 and SRA advance the phagocytosis of degenerated myelin by promoting the activation of paxillin and cofilin whereas SIRP $\alpha$ inhibits phagocytosis by promoting the inactivation of paxillin and cofilin (a schematic representation of the working hypothesis). Degenerated myelin ligates simultaneously phagocytic receptors CR3 and SRA and immune inhibitory receptor SIRP $\alpha$. CR3 and SRA produce the signaling that culminates in structural changes (marked by ellipses);

filopodia and lamellipodia first engulf the myelin-debris as they extend and then pull-in the myelin-debris as they retract. We hypothesize the following. Phagocytic receptors promote paxillin activation through phosphorylation (paxillin $\rightarrow$ p-paxillin) leading to cofilin activation through dephosphorylation (p-cofilin $\rightarrow$ cofilin). In turn, cofilin promotes the production of filopodia and lamellipodia and so phagocytosis is advanced. In contrast, SIRP $\alpha$ promotes the inactivation of paxillin through p-paxillin dephosphorylation (p-paxillin $\rightarrow$ paxillin) leading to cofilin inactivation through phosphorylation (cofilin $\rightarrow$ p-cofilin). In turn, the production of filopodia and lamellipodia is reduced and so phagocytosis is inhibited. Retraction of filopodia and lamellipodia is promoted by MLCK triggering motor activity in non-muscle-myosin which then initiates F-actin/non-muscle-myosin-based contraction (not illustrated). Dashed lines mark indirect interactions; blue lines mark activation and red lines inhibition of phagocytosis. as control microglia in text and Control Luciferase (Con-Luc) microglia in figures.

\section{MYELIN ISOLATION}

Myelin isolation from mouse brains has been previously described (Slobodov et al., 2001) and also visualized (Gitik et al., 2011). The isolated myelin is "degenerated myelin" since isolation involves breakdown of intact myelin.

\section{PHAGOCYTOSIS OF DEGENERATED MYELIN}

Phagocytosis of degenerated myelin was assayed in microglia that were plated in 96-well tissue culture plates at a density that minimizes cell-cell contact $\left(0.25-1.5 \times 10^{4} /\right.$ well $)$ in the presence of Dulbecco's Modified Eagle Medium (DMEM)/F12 supplemented by $10 \%$ FCS. Non-adherent microglia were washed out after $2 \mathrm{~h}$ and adherent microglia left to rest overnight. Next, phagocytes were washed and degenerated myelin added in the presence of serum for the indicated time periods. Then, unphagocytosed degenerated myelin was washed out and levels of phagocytosis determined by enzyme linked immunosobent assay (ELISA).

\section{ELISA ASSAY TO OUANTIFY THE PHAGOCYTOSIS OF DEGENERATED MYELIN}

ELISA assay to quantify the phagocytosis of degenerated myelin is based on the detection of myelin basic protein (MBP) in microglia lysates. Since MBP is unique to myelin and is not produced by phagocytes, MBP levels detected in phagocyte cytoplasm are proportional to levels of degenerated myelin that is phagocytosed. In brief, after non-phagocytosed degenerated myelin is washed away and remaining degenerated myelin has been phagocytosed/internalized, phagocytes are lysed $(0.05 \mathrm{M}$ carbonate buffer, $\mathrm{pH} 10$ ), lysates transferred to high protein absorbance plates (Nalge Nunc International, NY, USA) and levels of MBP determined by ELISA using anti-MBP monoclonal antibody. A detailed protocol is given in (Slobodov et al., 2001) where we also determined that more than $95 \%$ of the detected MBP arises from phagocytosed/internalized degenerated myelin. We further verified the validity of this phagocytosis assay by documenting over 95\% inhibition of the phagocytosis of degenerated myelin in the presence of cytochalasin-D (not shown).

\section{QUANTIFICATION OF PHAGOCYTOSIS}

Quantification of phagocytosis was carried out in the following way. When phagocytosis by SIRP $\alpha$-KD microglia (i.e., microglia in which SIRP $\alpha$ expression was knocked-down with SIRP $\alpha$-shRNA) was compared to phagocytosis by control microglia (i.e., microglia infected with non-target Luciferase-shRNA), phagocytosis by each population was first normalized to the number of respective microglia counted in $1 \mathrm{~mm}^{2}$ areas at the center of wells. Normalizing phagocytosis to cell number is required since SIRP $\alpha-\mathrm{KD}$ and control microglia may differ in their adherence properties, thus resulting in different number of adherent microglia even when the same number of cells was initially seeded. To this end, microglia in replicate plates were fixed (i.e., instead of being lysed for the phagocytosis assay), stained, and counted. Phagocytosis normalized to cell number by control microglia was defined $100 \%$ and phagocytosis normalized to cell number by SIRP $\alpha-K D$ 
microglia was calculated as percentage of phagocytosis by control microglia. Statistical analysis was carried out as detailed in figure legends.

\section{IMMUNOBLOT ANALYSIS}

Immunoblot analysis Microglia were plated in $10 \mathrm{~cm}$ tissue culture plates at a density that minimizes cell-cell contact $\left(3 \times 10^{6}\right.$ cells per plate) in the presence of DMEM supplemented by $10 \%$ FCS, and left to rest overnight. Phagocytes were washed in fresh DMEM supplemented by $10 \%$ FCS, degenerated myelin added in the presence of serum for the indicated time periods and unphagocytosed degenerated myelin washed out. For lysis, microglia were washed in PBS and lysed in ice cold lysis buffer (Tris HCL $1 \mathrm{M} \mathrm{pH} 7.5, \mathrm{MgCl}_{2} 1 \mathrm{M}, \mathrm{NaCl} 4 \mathrm{M}, 0.5 \% \mathrm{NP}-40$, $0.1 \% \mathrm{DTT}$, and $0.1 \% \mathrm{NaVa}$ ) supplemented with protease and phosphatase inhibitors cocktail (Sigma-Aldrich, Israel), cellular debris was removed by centrifugation, and total protein content determined using Bradford reagent (Sigma-Aldrich, Israel). Equal protein content from whole cell lysates was separated on SDS-PAGE to detect SIRP $\alpha$, paxillin, cofilin, and GAPDH. Proteins were blotted to nitrocellulose membranes, blocked with $10 \%$ non-fat milk or 5\% BSA in TBS (Tris-buffered saline) for $1 \mathrm{~h}$ at RT, incubated over night at $4^{\circ} \mathrm{C}$ in the presence of primary antibodies mouse anti-rat SIRP $\alpha / C D 172 \alpha$ (Serotec, Oxford, England), mouse anti-human GAPDH, rabbit anti-cofilin, rabbit anti-pS ${ }^{3}$-cofilin-1, and rabbit anti-pY ${ }^{118}$-paxillin (Santa Cruz Biotechnology, USA), rabbit anti-paxillin (Cell Signaling, USA), and mouse anti-actin monoclonal antibody (MP biomedicals, CA, USA). Blots were washed with TBST and incubated with respective secondary antibodies goat anti-rat, goat anti-rabbit, and goat antimouse conjugated to HRP (Jackson ImmunoReserach, USA) for 40-min at RT. Proteins were visualized with EZ-ECL kit for HRP detection (Beit Haemek, Israel). The intensities of immunoblot bands were determined by ImageJ software and quantification and statistical analysis was carried out as detailed in figure legends.

\section{RESULTS}

\section{SIRP $\alpha$ PROMOTES THE INACTIVATION OF COFILIN}

We previously documented the following with regard to the phagocytosis of degenerated myelin by microglia in the absence of anti-myelin antibodies: (a) phagocytosis is mostly mediated by CR3 and to a lesser degree by SRA (Rotshenker, 2003), (b) phagocytosis is advanced by cofilin (Hadas et al., 2012), (c) phagocytosis is inhibited by SIRP $\alpha$ (Gitik et al., 2011). Thus, SIRP $\alpha$ could inhibit phagocytosis by promoting the inactivation of cofilin. In this case, reducing SIRP $\alpha$ in phagocytes is expected to promote the activation of cofilin by shifting the balance from inactive $\mathrm{p}$-cofilin (cofilin phosphorylated at serine site $\mathrm{S}^{3}$ ) to active unphosphorylated cofilin (cofilin) concurrent with augmenting phagocytosis. We addressed this issue by examining p-cofilin levels before and during phagocytosis in microglia in which $\operatorname{SIRP} \alpha$ expression was knocked-down (SIRP $\alpha-K D)$ by lentiviral infection with SIRP $\alpha$ shRNA, and further, in control microglia that were similarly infected with the non-target firefly Luciferase-shRNA.

Indeed, SIRP $\alpha$ levels were reduced (Figure 2A) and phagocytosis was augmented (Figure 2B) in $\operatorname{SIRP} \alpha-K D$ microglia

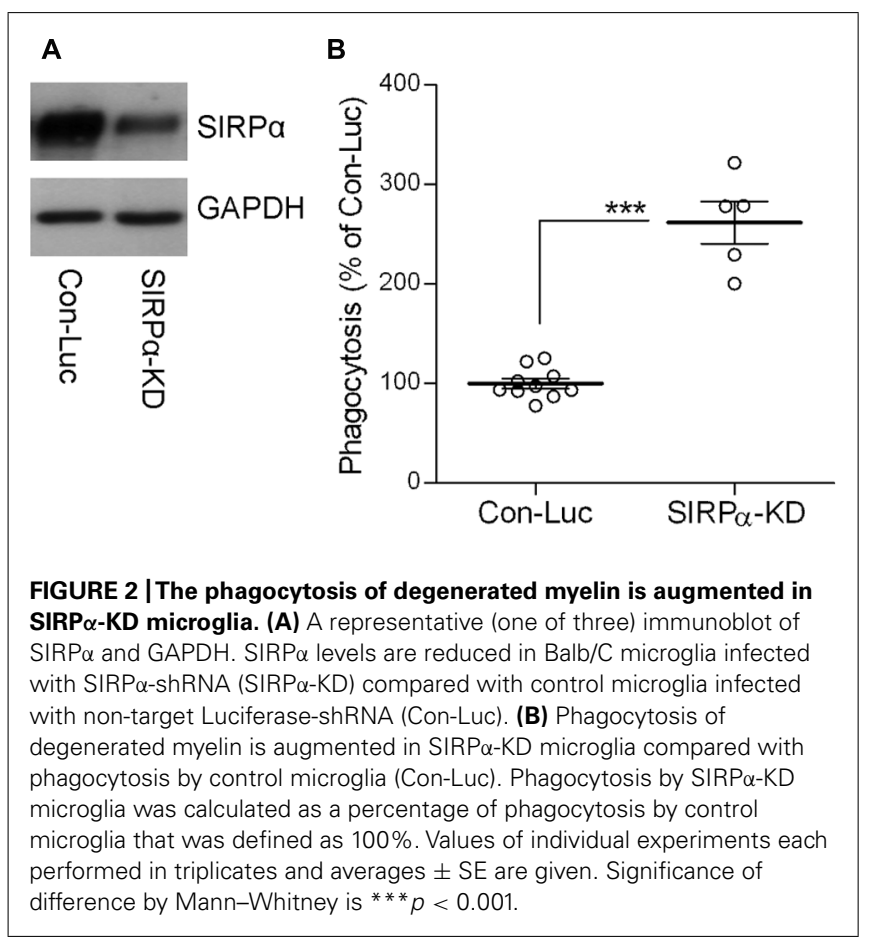

compared with control microglia, thus confirming our previously reported observations (Gitik etal., 2011). Levels of p-cofilin were then determined by immunoblot analysis using an antibody raised against cofilin that is phosphorylated at serine site $S^{3}$ (Figures 3A,C). Levels of p-cofilin were reduced in control microglia after $10 \mathrm{~min}$ of phagocytosis down to about $75 \%$ of those in non-phagocytosing control microglia. Then, after $30 \mathrm{~min}$ of phagocytosis, levels of p-cofilin returned to and rose above their initial levels in non-phagocytosing control microglia. These findings confirm our previously reported observations (Hadas et al., 2012).

SIRP $\alpha-K D$ microglia differed from control microglia with respect to $\mathrm{p}$-cofilin levels before and during phagocytosis (Figures 3B,C). Levels of p-cofilin were reduced in nonphagocytosing $\operatorname{SIRP} \alpha-\mathrm{KD}$ microglia down to about $75 \%$ of those in non-phagocytosing control microglia. After 10 and $30 \mathrm{~min}$ of phagocytosis, p-cofilin levels were reduced further down to about $60 \%$ of those in non-phagocytosing control microglia. Taken together, cofilin was transiently activated during prolonged phagocytosis in control microglia but continuously activated in SIRP $\alpha$-KD microglia, suggesting that normally SIRP $\alpha$ promotes the inactivation of cofilin through serine $\left(S^{3}\right)$ phosphorylation.

\section{SIRP $\alpha$ PROMOTES THE INACTIVATION OF PAXILLIN}

Taken that the SIRP $\alpha /$ SHP-1/2 complex dephosphorylates phosphotyrosine sites in its immediate target molecules (Barclay and Brown, 2006; Matozaki et al., 2009; Oldenborg, 2013) and our present findings that $\operatorname{SIRP} \alpha$ promotes cofilin inactivation by serine phosphorylation, $\operatorname{SIRP} \alpha$ could not have inactivated cofilin directly. However, $\operatorname{SIRP} \alpha$ could inactivate cofilin indirectly through paxillin. This proposition is based on previous observations that paxillin is activated by tyrosine phosphorylation 


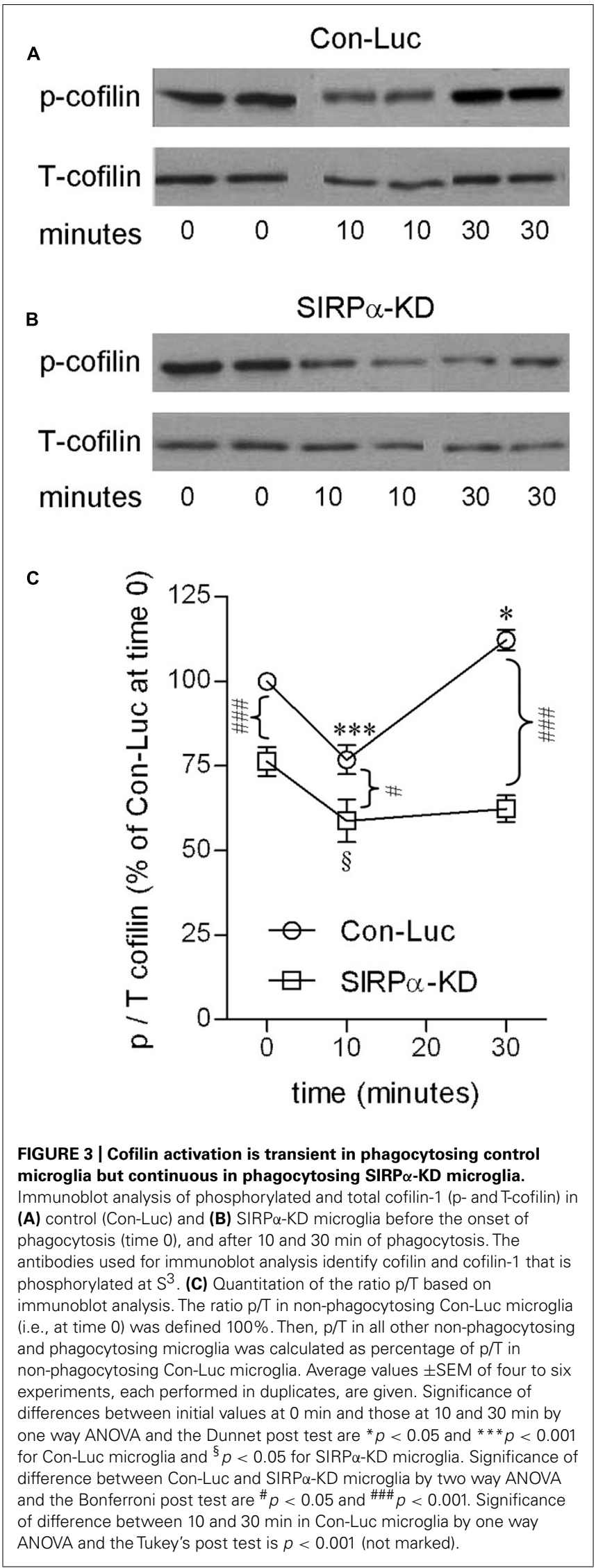

(paxillin phosphorylated at tyrosine site $\mathrm{Y}^{118}$ ), and further, that p-paxillin can indirectly activate cofilin (Deakin and Turner, 2008). Thus if SIRP $\alpha$ promotes the inactivation of paxillin, then levels of active p-paxillin are expected to be higher in SIRP $\alpha-\mathrm{KD}$ microglia than in control microglia.

Levels of p-paxillin were determined by immunoblot analysis using an antibody raised against paxillin which is phosphorylated at tyrosine site $\mathrm{Y}^{118}$ (Figures 4A,C). Levels of p-paxillin increased in control microglia to about $160 \%$ of those in non-phagocytosing control microglia after $10 \mathrm{~min}$ of phagocytosis. Then, after $30 \mathrm{~min}$ of phagocytosis, p-paxillin levels decreased significantly to about $120 \%$ of those in non-phagocytosing control microglia.

SIRP $\alpha-K D$ microglia differed from control microglia with respect to p-paxillin levels before and during phagocytosis (Figures 4B,C). Levels of p-paxillin were about $145 \%$ higher in non-phagocytosing SIRP $\alpha-K D$ microglia than in nonphagocytosing control microglia. After 10 and $30 \mathrm{~min}$ of phagocytosis, levels of p-paxillin increased further to about 160 and $170 \%$ of those in non-phagocytosing control microglia. Taken together, paxillin was transiently activated during prolonged phagocytosis in control microglia and continuously activated in SIRP $\alpha$-KD microglia, suggesting that normally SIRP $\alpha$ promotes the inactivation of paxillin through phosphotyrosine $\left(\mathrm{pY}^{118}\right)$ dephosphorylation.

\section{PAXILLIN ACTIVATION, COFILIN ACTIVATION AND PHAGOCYTOSIS POSITIVELY CORRELATE WITH ONE ANOTHER}

It has been suggested that active p-paxillin can indirectly activate cofilin by promoting the transition from inactive $\mathrm{p}$-cofilin to active unphosphorylated cofilin (Deakin and Turner, 2008). If this is the case during the phagocytosis of degenerated myelin, then levels of active p-paxillin and levels of inactive p-cofilin should negatively correlate with one another during phagocytosis, and further, phagocytosis augmentation should positively correlate with the activation of both paxillin and cofilin. Indeed, control microglia displayed transient increases in p-paxillin and transient decreases in $\mathrm{p}$-cofilin during phagocytosis, and further, SIRP $\alpha-\mathrm{KD}$ microglia exhibited higher levels of p-paxillin and lower levels of p-cofilin compared with control microglia both before and throughout phagocytosis which was augmented in SIRP $\alpha-\mathrm{KD}$ microglia compared with control microglia. To obtain a quantitative measure to these apparent correlations, all values of p-paxillin and p-cofilin presented in Figures $\mathbf{3}$ and $\mathbf{4}$ were subjected to linear regression and correlation analysis (Figure 5). Levels of p-paxillin and pcofilin displayed significant $(p<0.05)$ negative correlation with an $r^{2}$ value of 0.79 . Thus paxillin activation (reflected by higher levels of p-paxillin) and cofilin activation (reflected by lower levels of p-cofilin) positively correlated with one another. Since levels of phagocytosis increased in SIRP $\alpha$-KD microglia compared with control microglia at the same time as levels of both paxillin and cofilin activation increased, all three (i.e., levels of phagocytosis, paxillin activation, and cofilin activation) positively correlated with one another.

\section{DISCUSSION}

Observations made in this study suggest a novel mechanism whereby paxillin and cofilin are targeted to control the 
A

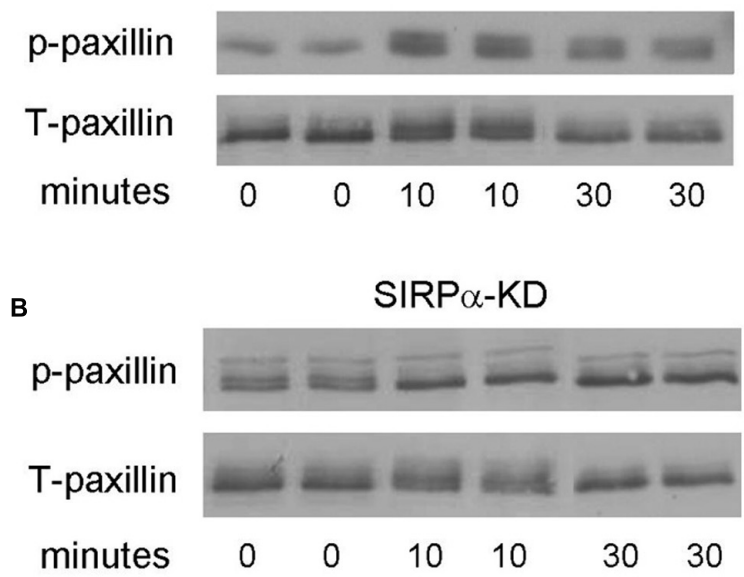

C

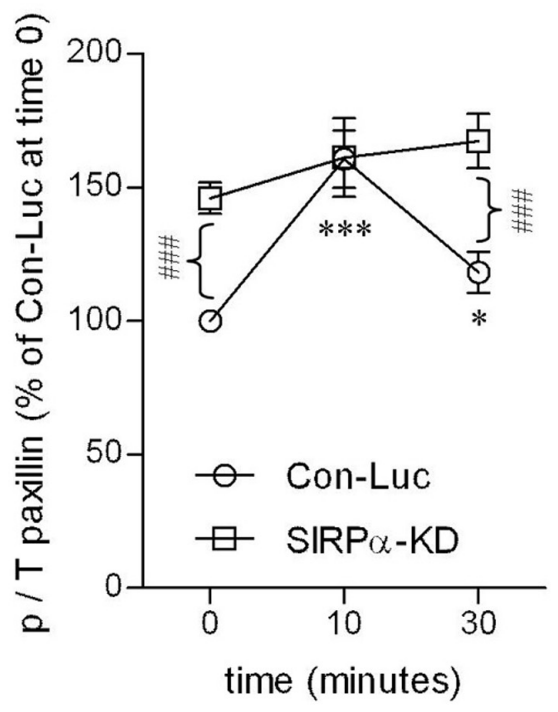

FIGURE 4 | Paxillin activation is transient in phagocytosing control microglia but continuous in phagocytosing SIRP $\alpha-K D$ microglia. Immunoblot analysis of $\mathrm{p}$ - and T-paxillin in (A) control (Con-Luc), and (B) SIRP $\alpha-K D$ microglia before the onset of phagocytosis (time 0 ), and after 10 and 30 min of phagocytosis. The antibodies used for immunoblot analysis identify paxillin and paxillin that is phosphorylated at $Y^{118}$. (C) Quantitation of the ratio $p / T$ based on immunoblot analysis. The ratio $p / T$ in non-phagocytosing Con-Luc microglia (i.e., at time 0 ) was defined $100 \%$. Then $\mathrm{p} / \mathrm{T}$ in all other non-phagocytosing and phagocytosing microglia was calculated as percentage of $\mathrm{p} / \mathrm{T}$ in non-phagocytosing Con-Luc microglia. Average values $\pm S E M$ of four to eight experiments, each performed in duplicates, are given. Significance of differences between initial values at 0 min and those at 10 and 30 min by one way ANOVA and the Dunnet post test are ${ }^{*} p<0.05$ and ${ }^{* *} p<0.001$ for Con-Luc microglia. Significance of difference between Con-Luc and SIRP $\alpha$-KD microglia by two way ANOVA and the Bonferroni post test is $\# \# p<0.001$. Significance of difference between 10 and $30 \mathrm{~min}$ in Con-Luc microglia by one way ANOVA and the Tukey's post test is $p<0.01$ (not marked).

phagocytosis of degenerated myelin by both the activating signaling which phagocytic receptors CR3 and SRA produce and the inhibiting signaling that immune inhibitory receptor $\mathrm{SIRP} \alpha$ produces. In this regard, phagocytic receptors advance

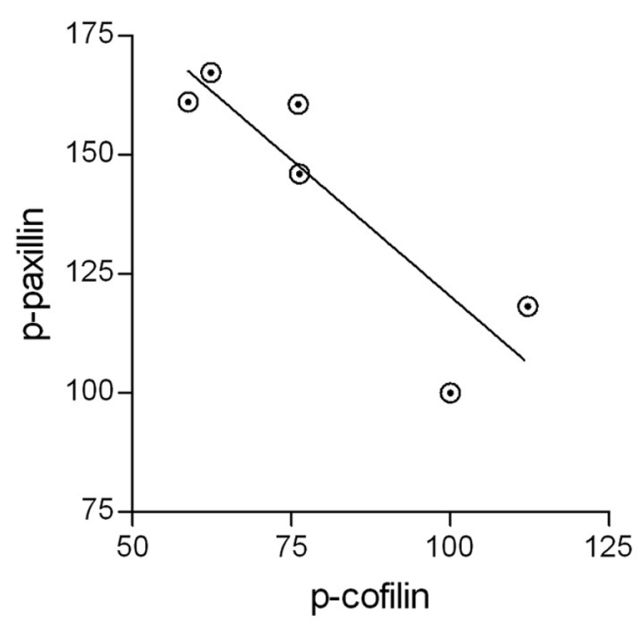

FIGURE 5 | The activation of paxillin and cofilin are linked. All values of active p-paxillin and inactive p-cofilin that are presented in Figures $\mathbf{3}$ and $\mathbf{4}$ were subjected to linear regression and correlation analysis. The two display a negative correlation with an $r^{2}$ value of 0.79 . Significance of correlation by the Pearson double tailed test is $p<0.05$.

phagocytosis by promoting the activation of paxillin and cofilin whereas immune inhibitory receptor SIRP $\alpha$ inhibits phagocytosis by promoting the inactivation of the two.

The activation of phagocytosis by paxillin and cofilin is suggested by the findings that phagocytosis positively correlates with the activation of both paxillin and cofilin in both control and SIRP $\alpha-K D$ microglia. In control microglia, paxillin and cofilin were activated concurrent with the activation of phagocytosis, and in SIRP $\alpha-K D$ microglia, levels of paxillin and cofilin activation increased simultaneously with the increase in phagocytosis.

The role cofilin plays in the phagocytosis of degenerated myelin was recently reported and discussed by us (Hadas et al., 2012). Cofilin advances phagocytosis by promoting the remodeling of Factin and thereby the production of filopodia/lamellipodia which engulf the myelin-debris. We presently suggest that cofilin activation is promoted by paxillin. This proposition is based on the observations that paxillin can indirectly activate cofilin (Deakin and Turner, 2008) and our present findings that levels of paxillin activation and levels of cofilin activation positively correlated with one another. The involvement of p-paxillin in signaling phagocytosis by CR3 and SRA which is documented here could also take place in signaling phagocytosis by $\mathrm{Fc} \gamma \mathrm{R}$ since paxillin is tyrosine phosphorylated during $\mathrm{F} c \gamma \mathrm{R}$ mediated phagocytosis (Greenberg et al., 1994).

That SIRP $\alpha$ inhibits the phagocytosis of degenerated myelin by promoting the inactivation of both paxillin and cofilin is suggested by the synchronized occurrence of three events in SIRP $\alpha$ $\mathrm{KD}$ microglia. First, phagocytosis is augmented in SIRP $\alpha-\mathrm{KD}$ microglia compared with control microglia; second, the activation of both paxillin and cofilin increases in non-phagocytosing as well as in phagocytosing $\operatorname{SIRP} \alpha-\mathrm{KD}$ microglia compared with non-phagocytosing and phagocytosing control microglia; third, the kinetics of the activation of both paxillin and cofilin switched from transient in phagocytosing control microglia to continuous 
in phagocytosing SIRP $\alpha-K D$ microglia. SIRP $\alpha$ dependent inactivation of paxillin and cofilin was not reported before for any of the functions that SIRP $\alpha$ is involved in. We further raise the possibility that SIRP $\alpha$ could directly inactivate paxillin through dephosphorylation since paxillin is activated through tyrosine phosphorylation (Deakin and Turner, 2008) and the SIRP $\alpha /$ SHP1/2 complex dephosphorylates phosphotyrosine sites (Barclay and Brown, 2006; Matozaki et al., 2009; Oldenborg, 2013).

In conclusion, the phagocytosis of degenerated myelin is determined by the balance between the signaling produced by CR3 and SRA which activates paxillin and cofilin and the signaling produced by SIRP $\alpha$ which inactivates paxillin and cofilin. SIRP $\alpha$ could also target paxillin and cofilin while inhibiting phagocytosis which is mediated by additional phagocytic receptors such as $\mathrm{Fc} \gamma \mathrm{R}$ (Oldenborg et al., 2001) and during the phagocytosis of particles other than myelin-debris such as aging red blood cells (Oldenborg et al., 2000) and tumor cells (Chao et al., 2012; Kim et al., 2012).

\section{ACKNOWLEDGMENT}

This work was supported by grant Number 11/06 from the Israel Science Foundation.

\section{REFERENCES}

Barclay, A. N., and Brown, M. H. (2006). The SIRP family of receptors and immune regulation. Nat. Rev. Immunol. 6, 457-464. doi: 10.1038/nri1859

Bernstein, B. W., and Bamburg, J. R. (2010). ADF/cofilin: a functional node in cell biology. Trends Cell Biol. 20, 187-195. doi: 10.1016/j.tcb.2010.01.001

Chao, M. P., Weissman, I. L., and Majeti, R. (2012). The CD47-SIRPalpha pathway in cancer immune evasion and potential therapeutic implications. Curr. Opin. Immunol. 24, 225-232. doi: 10.1016/j.coi.2012.01.010

Clark, K., Langeslag, M., Figdor, C. G., and van Leeuwen, F. N. (2007). Myosin II and mechanotransduction: a balancing act. Trends Cell Biol. 17, 178-186. doi: 10.1016/j.tcb.2007.02.002

David, S., and Aguayo, A. J. (1981). Axonal elongation into peripheral nervous system "bridges" after central nervous system injury in adult rats. Science 214, 931-933. doi: 10.1126/science.6171034

Deakin, N. O., and Turner, C. E. (2008). Paxillin comes of age. J. Cell Sci. 121, 2435-2444. doi: 10.1242/jcs.018044

Gitik, M., Liraz Zaltsman, S., Oldenborg, P. A., Reichert, F., and Rotshenker, S. (2011). Myelin down-regulates myelin phagocytosis by microglia and macrophages through interactions between CD47 on myelin and SIRPalpha (signal regulatory protein-alpha) on phagocytes. J. Neuroinflammation 8,24 . doi: 10.1186/1742-2094-8-24

Gitik, M., Reichert, F., and Rotshenker, S. (2010). Cytoskeleton plays a dual role of activation and inhibition in myelin and zymosan phagocytosis by microglia. FASEB J. 24, 2211-2221. doi: 10.1096/fj.09-146118

Greenberg, S., Chang, P., and Silverstein, S. C. (1994). Tyrosine phosphorylation of the gamma subunit of Fc gamma receptors, p72syk, and paxillin during Fc receptor-mediated phagocytosis in macrophages. J. Biol. Chem. 269, 3897-3902.

Hadas, S., Spira, M., Hanisch, U. K., Reichert, F., and Rotshenker, S. (2012). Complement receptor-3 negatively regulates the phagocytosis of degenerated myelin through tyrosine kinase Syk and cofilin. J. Neuroinflammation 9, 166. doi: 10.1186/1742-2094-9-166

Han, M. H., Lundgren, D. H., Jaiswal, S., Chao, M., Graham, K. L., Garris, C. S., et al. (2012). Janus-like opposing roles of CD47 in autoimmune brain inflammation in humans and mice. J. Exp. Med. 209, 1325-1334. doi: 10.1084/jem.201 01974

Kim, D., Wang, J., Willingham, S. B., Martin, R., Wernig, G., and Weissman, I. L. (2012). Anti-CD47 antibodies promote phagocytosis and inhibit the growth of human myeloma cells. Leukemia 26, 2538-2545. doi: 10.1038/leu. 2012.141

Kotter, M. R., Li, W. W., Zhao, C., and Franklin, R. J. M. (2006). Myelin impairs CNS remyelination by inhibiting oligodendrocyte precursor cell differentiation. J. Neurosci. 26, 328-332. doi: 10.1523/JNEUROSCI.2615-05.2006
Matozaki, T., Murata, Y., Okazawa, H., and Ohnishi, H. (2009). Functions and molecular mechanisms of the CD47-SIRPalpha signaling pathway. Trends Cell Biol. 19, 72-80. doi: 10.1016/j.tcb.2008.12.001

Mead, R. J., Singhrao, S. K., Neal, J. W., Lassmann, H., and Morgan, B. P. (2002). The membrane attack complex of complement causes severe demyelination associated with acute axonal injury. J. Immunol. 168, 458-465.

Oldenborg, P. A. (2013). CD47: a cell surface glycoprotein which regulates multiple functions of hematopoietic cells in health and disease. ISRN Hematol. 2013, 614619. doi: 10.1155/2013/614619

Oldenborg, P. A., Gresham, H. D., and Lindberg, F. P. (2001). CD47-signal regulatory protein alpha (SIRPalpha) regulates Fcgamma and complement receptor-mediated phagocytosis. J. Exp. Med. 193, 855-862. doi: 10.1084/jem.193. 7.855

Oldenborg, P. A., Zheleznyak, A., Fang, Y. F., Lagenaur, C. F., Gresham, H. D., and Lindberg, F. P. (2000). Role of CD47 as a marker of self on red blood cells. Science 288, 2051-2054. doi: 10.1126/science.288.5473.2051

Reichert, F., and Rotshenker, S. (1996). Deficient activation of microglia during optic nerve degeneration. J. Neuroimmunol. 70, 153-161. doi: 10.1016/S01655728(96)00112-9

Reichert, F., and Rotshenker, S. (1999). Galectin-3/MAC-2 in experimental allergic encephalomyelitis. Exp. Neurol. 160, 508-514. doi: 10.1006/exnr.1999.7229

Reichert, F., and Rotshenker, S. (2003). Complement-receptor-3 and scavengerreceptor-AI/II mediated myelin phagocytosis in microglia and macrophages. Neurobiol. Dis. 12, 65-72. doi: 10.1016/S0969-9961(02)00008-6

Reichert, F., Slobodov, U., Makranz, C., and Rotshenker, S. (2001). Modulation (inhibition and augmentation) of complement receptor-3- mediated myelin phagocytosis. Neurobiol. Dis. 8, 504-512. doi: 10.1006/nbdi.2001. 0383

Rotshenker, S. (2003). Microglia and macrophage activation and the regulation of complement-receptor-3 (CR3/MAC-1)-mediated myelin phagocytosis in injury and disease. J. Mol. Neurosci. 21, 65-72. doi: 10.1385/JMN:21:1:65

Rotshenker, S. (2011). Wallerian degeneration: the innate immune response to traumatic nerve injury. J. Neuroinflammation 8, 109. doi: 10.1186/1742-2094-8109

Slobodov, U., Reichert, F., Mirski, R., and Rotshenker, S. (2001). Distinct inflammatory stimuli induce different patterns of myelin phagocytosis and degradation in recruited macrophages. Exp. Neurol. 167, 401-409. doi: 10.1006/exnr.2000.7559

Stadelmann, C., and Bruck, W. (2008). Interplay between mechanisms of damage and repair in multiple sclerosis. J. Neurol. 255 (Suppl. 1), 12-18. doi 10.1007/s00415-008-1003-7

Vargas, M. E., and Barres, B. A. (2007). Why is Wallerian degeneration in the CNS so slow? Annu. Rev. Neurosci. 30, 153-179. doi: 10.1146/annurev.neuro.30.051606.094354

Vicente-Manzanares, M., Ma, X., Adelstein, R. S., and Horwitz, A. R. (2009). Nonmuscle myosin II takes centre stage in cell adhesion and migration. Nat. Rev. Mol.Cell Biol. 10, 778-790. doi: 10.1038/nrm2786

Waller, A. (1850). Experiments on the section of the glossopharyngeal and hypoglossal nerves of the frog, observations of the alterations produced thereby in the structure of their primitive fibers. Phil. Transact. Royal. Soc. London 140, 423-429. doi: 10.1098/rstl.1850.0021

Wang, W., Eddy, R., and Condeelis, J. (2007). The cofilin pathway in breast cancer invasion and metastasis. Nat. Rev. Cancer 7, 429-440. doi: 10.1038/nrc2148

Conflict of Interest Statement: The authors declare that the research was conducted in the absence of any commercial or financial relationships that could be construed as a potential conflict of interest.

Received: 26 February 2014; accepted: 24 March 2014; published online: 16 April 2014. Citation: Gitik M, Kleinhaus R, Hadas S, Reichert Fand Rotshenker S (2014) Phagocytic receptors activate and immune inhibitory receptor SIRP $\alpha$ inhibits phagocytosis through paxillin and cofilin. Front. Cell. Neurosci. 8:104. doi: 10.3389/fncel.2014.00104 This article was submitted to the journal Frontiers in Cellular Neuroscience. Copyright (C) 2014 Gitik, Kleinhaus, Hadas, Reichert and Rotshenker. This is an openaccess article distributed under the terms of the Creative Commons Attribution License (CC BY). The use, distribution or reproduction in other forums is permitted, provided the original author(s) or licensor are credited and that the original publication in this journal is cited, in accordance with accepted academic practice. No use, distribution or reproduction is permitted which does not comply with these terms. 